\title{
Enzymes Photocrosslinked to Live Cell Receptors Retain Activity and EGFR Inhibition After Both Internalization and Recycling
}

Shambojit Roy, ${ }^{1}$ Michael Brasino, ${ }^{1, \dagger}$ Jonathan M. Beirne, ${ }^{2}$ Albert Harguindey, ${ }^{1}$ Douglas A. Chapnick, ${ }^{2}$ Xuedong Liu, ${ }^{2}$ Jennifer N. Cha, ${ }^{*, 1,3}$ and Andrew P. Goodwin*,1,3

${ }^{1}$ Department of Chemical and Biological Engineering, ${ }^{2}$ Department of Biochemistry, ${ }^{3}$ Material Science and Engineering Program, University of Colorado, 596 UCB, Boulder, Colorado 80303, United States.

\section{Supplementary Information}

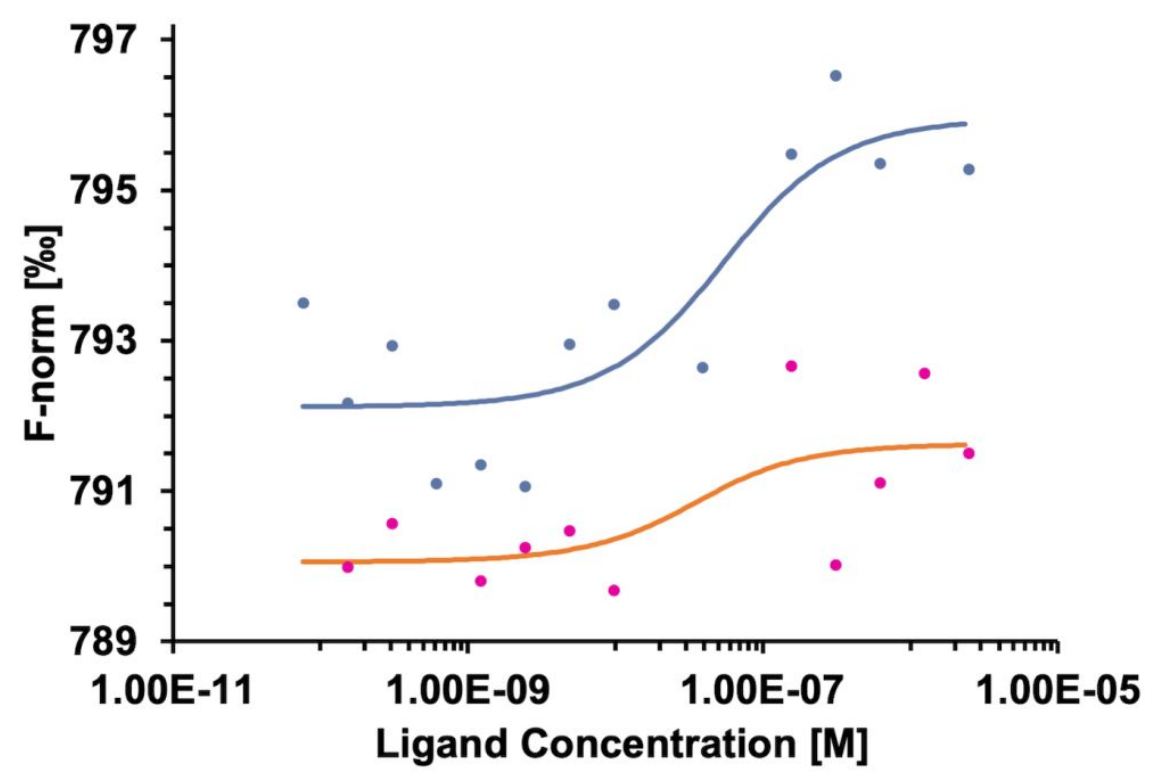

Figure S1. Comparison of microscale thermophoresis (MST) signal vs. affibody concentration for both N23BP-CodA (blue) and WT-CodA (orange) affibody-enzymes against $10 \mathrm{nM}$ of extracellular purified human EGFR, labeled with Alexa Fluor 647. 


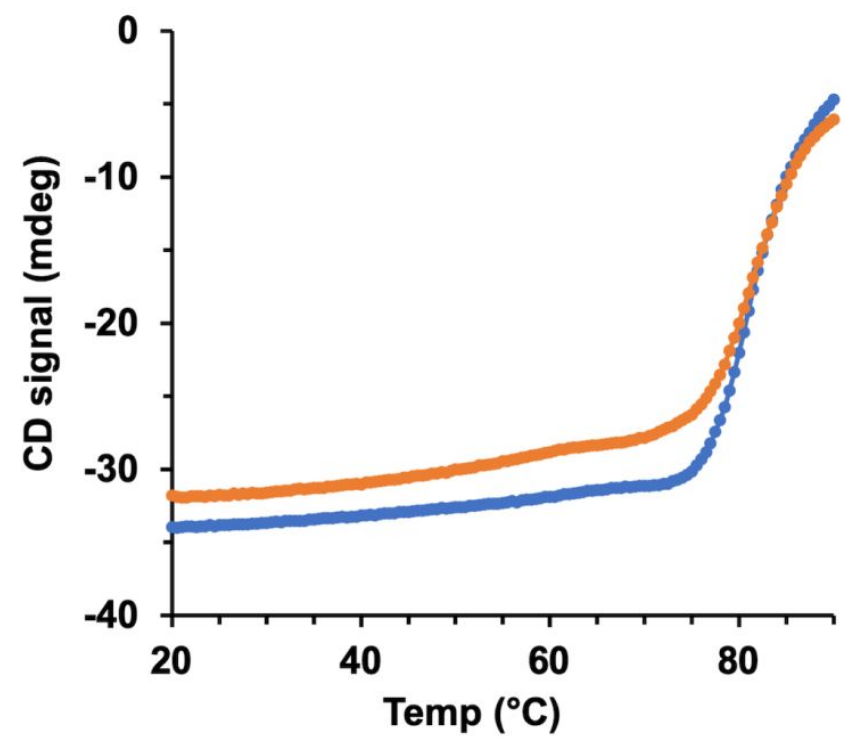

Figure S2. Melting curves showing circular dichroism at $222 \mathrm{~nm}$ vs. temperature for both N23BP-CodA (blue) and WT-CodA (orange) affibody-enzyme fusion proteins.

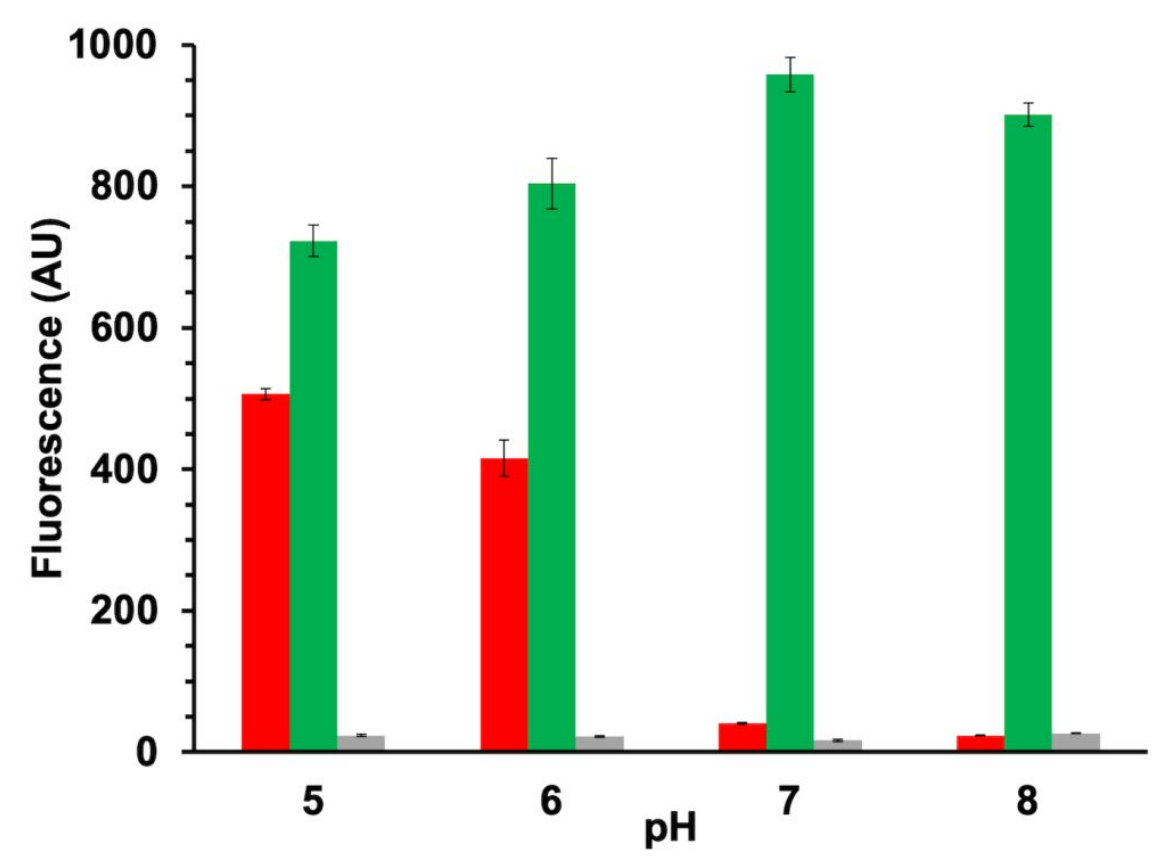

Figure S3. Comparison of fluorescence of $\mathrm{pHAb}$ ( $\mathrm{pH}$-dependent, red) and AF488 ( $\mathrm{pH}$ independent, green) dyes at different $\mathrm{pH}$; blank PBS (gray) is shown for comparison. Error bars represent standard error from three measurements. 

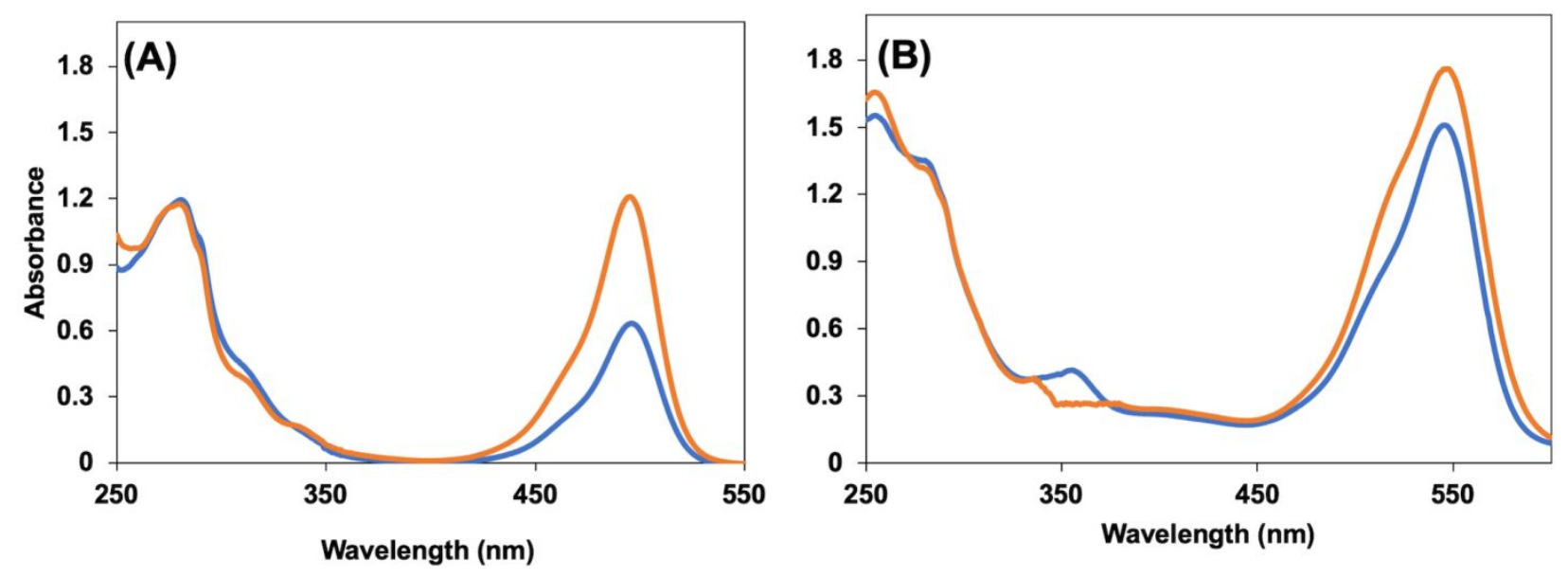

Figure S4. UV-Vis spectra for N23BP-CodA (blue) and WT-CodA (orange) conjugated to 10X molar excess of (a) NHS-AF488 and (b) NHS-pHAb.
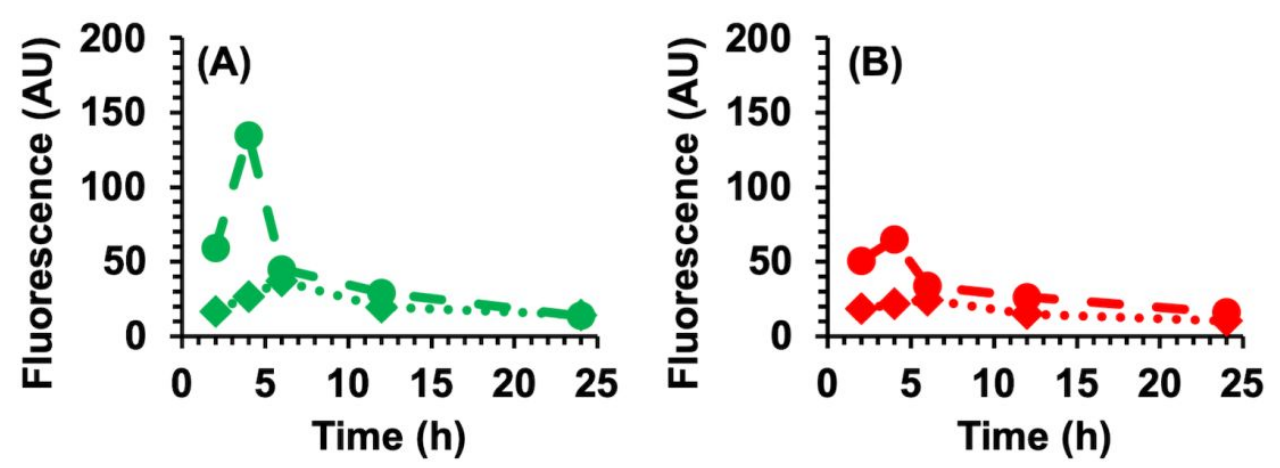

Figure S5. Change of mean fluorescent intensity with time of (A) AF488 and (B) conjugated to WTCodA (dashed line) and WT-CodA with free EGF added (dotted line). 

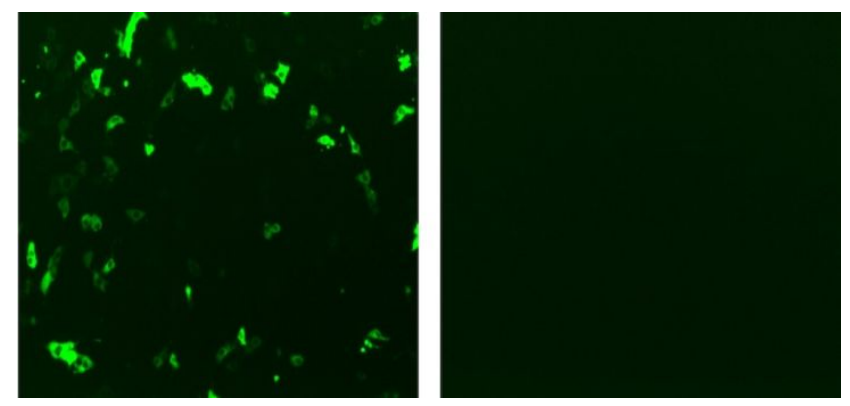

Figure S6. Fluorescence microscopy images comparing (left) EKAR-transfected and (right) nontransfected cells. The green fluorescence from the cells from the EKAR indicates successful transfection.

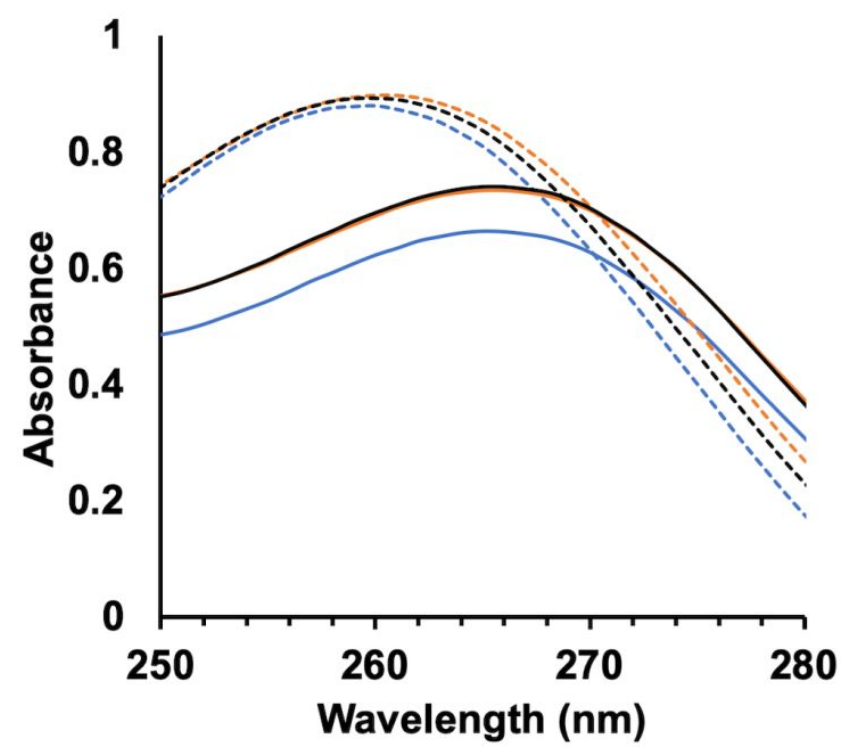

Figure S7. Absorbance spectra showing the conversion of cytosine (267 nm) to uracil (260 nm) before (solid) and $15 \mathrm{~min}$ after addition (dotted lines) of $50 \mathrm{nM} \mathrm{N23BP-CodA} \mathrm{(blue),} \mathrm{WT-CodA} \mathrm{(orange),} \mathrm{and}$ CodA (black) to $200 \mu \mathrm{M}$ of cytosine. 


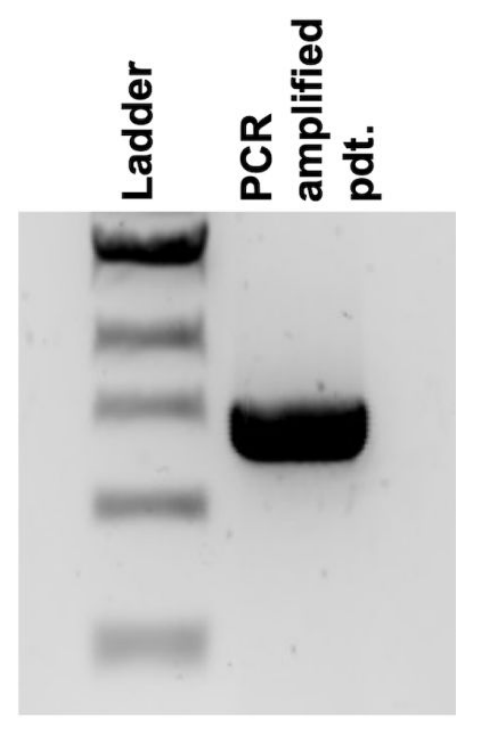

Figure S8. CodA gene (1.2 kbp) successfully amplified from E.Coli BL21(DE3) strain by PCR amplification. Ladder proteins are (top to bottom) 3, 2, 1.5, 1.0 and $0.5 \mathrm{kbp}$.

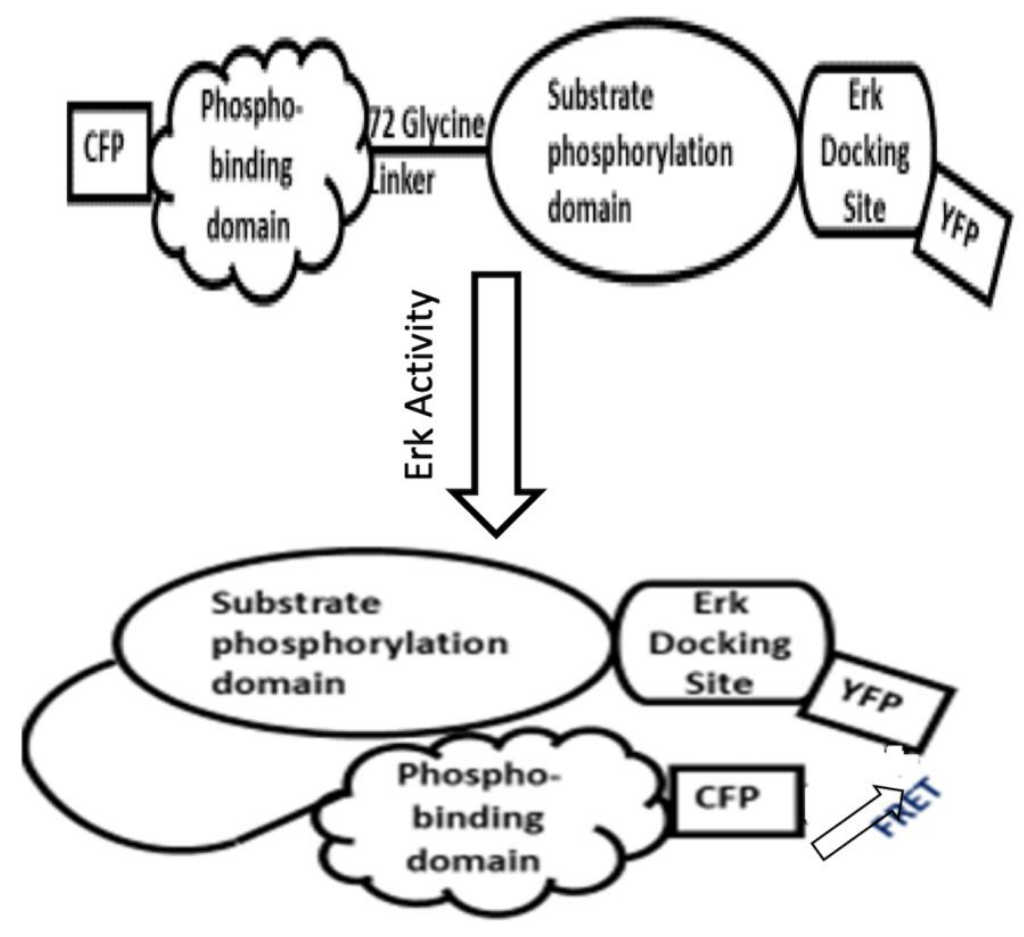

Figure S9. The schematic of the EKAR sensor. The ERK phosphorylation brings about a conformational change in the structure that result in the FRET. 
Table S1. Kinetic data for different enzyme fusion proteins.

\begin{tabular}{|l|l|l|l|}
\hline & CodA & N23BP-CodA & WT-CodA \\
\hline $\mathrm{Km}(\mathrm{mM})$ & 2.52 & 2.20 & 1.74 \\
\hline $\mathrm{kcat}\left(\mathrm{s}^{-1}\right)$ & 53.57 & 45.98 & 48.58 \\
\hline $\mathrm{kcat} / \mathrm{Km}\left(\mathrm{mM}^{-1} \mathrm{~s}^{-1}\right)$ & 21.23 & 20.89 & 27.90 \\
\hline
\end{tabular}

